\title{
Coarctation or interruption of aorta proximal to origin of both subclavian arteries Report of three cases presenting in infancy
}

\author{
A. R. Subramanian ${ }^{1,2}$ \\ From the Department of Cardiology, Royal Children's Hospital, Melbourne, Australia
}

Three cases of coarctation or interruption of the aorta proximal to the origin of both subclavian arteries in infancy are described. The characteristic clinical feature is the detection of good carotid and temporal pulses in association with poor brachial and femoral pulses. $A$ case is made for careful examination of all the peripheral pulses whenever the limb pulses are weak or absent. The rarity of the anomaly and the difficulty in the diagnosis of this condition in an infant with cardiac failure are emphasized.

Coarctation or interruption of the aorta proximal to the origin of both subclavian arteries is extremely rare. Only 4 cases have been reported so far, one in a 6-year-old girl who was operated successfully (Brynolf, Crafoord, and Mannheimer, 1958) and the other 3 in infancy (McNamara and Rosenberg, 1968). This report describes 3 additional cases with this anomaly presenting in early infancy. Despite the rarity of this condition, strangely enough these cases were all encountered within a period of six months.

\section{Case reports}

Case I A 4-day-old infant was admitted to hospital with a history of poor colour and respiratory difficulty since the day after birth. The infant was tachypnoeic with peripheral cyanosis and - poor limb pulses. The temporal and carotid pulses were readily felt. There was a moderately loud systolic murmur at the left sternal edge with a gallop rhythm. The liver was enlarged $5 \mathrm{~cm}$ below the costal margin. The lungs were clear. $X$-ray of the chest showed conspicuous cardiomegaly, with minimal lung plethora. An electrocardiogram showed right axis deviation with inversion of $T$ - wave in $V_{4} R$ and a $3 \mathrm{~mm} P$ wave in standard lead II. Cardiac catheterization and angiographic study revealed a juxtaductal coarctation of aorta, persistent ductus arteriosus, and ventricular septal defect with high pulmonary artery pressure. The subclavian arteries were not visualized and were presumed to be obstructed, or anomalous in origin. The infant was operated upon a week $\rightarrow$

1 Colombo Plan Fellow.

2 Permanent address: C/o Director General Armed Forces Medical Services, Ministry of Defence, New $=$ Delhi I I, India.

Received 3 December 1971. after admission when the segment of the aorta between the left common carotid artery and the ductus arteriosus was found to be absent and both the subclavian arteries were noticed to be arising anomalously beyond the ductus from the descending aorta. The interrupted aortic arch was repaired by using a Dacron bypass graft between the left common carotid artery and the distal aorta. The pulmonary artery was banded and the ductus was ligated. The patient, however, failed to improve and died on the ninth postoperative day. Death was attributed to cardiac failure.

Case 2 A ro-day-old infant was admitted to hospital with respiratory difficulty since birth and poor feeding of two days' duration. There was conspicuous tachycardia and tachypnoea with poor pulses, but the temporal pulses were found to be more readily felt than the brachials or femorals. There was a soft systolic murmur at the left sternal edge. The lungs were clear. The liver was enlarged $5 \mathrm{~cm}$ below costal margin. There was slight ankle oedema. With the treatment of cardiac failure, there was an improvement in the volume of all the pulses, and a difference between the temporal and the limb pulses became obvious. Chest $x$-ray showed cardiomegaly with some pulmonary congestion. Electrocardiogram revealed right axis deviation with right ventricular preponderance.

Cardiac catheterization and angiography confirmed the diagnosis of coarctation of the aorta proximal to the origin of both subclavian arteries. The child was operated upon 5 days after admission. At operation a preductal aortic coarctation with a confluence of vessels at the site of entry of the ductus was found. The vessels were identified as the two subclavian arteries and a persistent ductus arteriosus. The right subclavian artery was arising posteriorly and passed to the right 
side behind the oesophagus. The coarctation was resected and the ends of the aorta were anastomosed. The left subclavian artery was ligated and divided as it was very close to the site of coarctation. The ductus was also ligated and divided. The right subclavian artery was spared. Postoperatively the infant made a good recovery and remains asymptomatic.

Case 3 A ro-day-old baby was admitted to hospital with a history of grunting respiration, sweating, and poor feeding since the previous day. The infant was tachypnoeic with conspicuous tachycardia. All the pulses were of poor volume. Examination of the heart revealed a right ventricular impulse and a soft systolic murmur at the upper left sternal margin with a parasternal ejection click. The second sound was normal and no diastolic murmur was heard. The lungs were clear. The liver was $2 \mathrm{~cm}$ below costal margin. Chest $x$ ray showed slight cardiomegaly with mild pulmonary plethora and a prominent thymic shadow. Electrocardiogram revealed right axis deviation and right ventricular hypertrophy (upright $T$ in aVR, $V_{4} R, V$, and $V_{2}$ ). There was a diminutive $R$ with VS pattern in V5 and V6. A clinical diagnosis of hypoplastic left heart syndrome (Noonan and Nadas, I958; Gasul, Arcilla, and Lev, 1966) was tentatively made, and the baby was actively treated for cardiac failure pending investigation. The pulses improved after treatment, and on the fourth day after admission it was found that the brachials were still weak, but the temporals were readily felt. This suggested the diagnosis of coarctation of the aorta with both subclavian arteries arising beyond the site of coarctation. The diagnosis was subsequently confirmed by cardiac catheterization and angiography. The baby has further improved with medical treatment since then, and is maintaining satisfactory progress. An operation is therefore not being contemplated for the time being.

\section{Discussion}

Apart from the surgical aspects, this condition is of particular interest as it poses a diagnostic problem in infancy. Since both the subclavian arteries arise beyond the coarctation, the characteristic difference between the pulses in the upper and lower limbs is not observed.
Both the brachials and femoral pulses are weak or absent, but the carotids and temporals are usually felt quite readily. Unless one is aware of such a possibility and is in the habit of routinely palpating the carotid and temporal arteries whenever the limb pulses are poor, the condition is likely to be missed or misdiagnosed. The difficulty in diagnosis is often aggravated by the presence of cardiac failure with low cardiac output which tends to diminish the carotid and temporal pulses as well. This can be quite misleading and may result in a diagnosis of either aortic stenosis or hypoplastic left heart syndrome. The situation, however, becomes clear once the cardiac failure is brought under control. The cases described above exemplify the clinical picture and the diagnostic problem involved.

Case $I$ is quite clearly a case with interruption of the aortic arch. Case 2 is like a true coarctation, and in Case 3 it is impossible to say which type of lesion is present, because there has been no operation or necropsy proof.

I wish to thank Dr. A. W. Venables, Director, Department of Cardiology, and Mr. C. P. Clarke, Senior Cardiac Surgeon, Royal Children's Hospital, Melbourne, for their kind permission to publish these cases.

\section{References}

Brynolf, I., Crafoord, C., and Mannheimer, E. (1958). Coarctation of the aorta proximal to both subclavian arteries: case report of a six year old girl. fournal of Thoracic Surgery, 35, 123.

Gasul, B. M., Arcilla, R. A., and Lev, M. (1966). Heart Disease in Children - Diagnosis and Treatment. J. B. Lippincott, Philadelphia and Montreal.

McNamara, G., and Rosenberg, H. S. (1968). Interruption of aortic arch. In Paediatric Cardiology, p. 224. Ed. by Hamish Watson. Lloyd-Luke (Medical Books), London.

Noonan, J. A., and Nadas, A. S. (1958). The hypoplastic left heart syndrome. Pediatric Clinics of North America, 5, 1029.

Requests for reprints to Dr. A. W. Venables, Department of Cardiology, Royal Children's Hospital, Melbourne, Australia, 3052 